\title{
Analytical Calculation of a Class of Integrals Containing Exponential and Trigonometric Functions
}

\author{
By Vittorio Massidda
}

\begin{abstract}
It is shown how to evaluate analytically integrals from 0 to $2 \pi$ of functions of the type $f(\phi) g(\phi) \exp \{G(\phi)\}$, where $g(\phi)$ and $G(\phi)$ are linear combinations of powers of $\sin \phi$ and $\cos \phi$, and $f(\phi)$ is such that its Fourier coefficients can be evaluated analytically. The result is expressed in terms of modified Bessel functions.
\end{abstract}

\section{Introduction. Integrals of the type}

$$
\int_{0}^{2 \pi} \cos ^{n^{\prime}} \phi \sin ^{n^{\prime \prime}} \phi \exp \left\{\cos ^{m}\left(\phi-\phi_{0}\right)\right\} d \phi
$$

appear when evaluating the angular average of tensorial quantities whose distribution function is $\exp \left\{\cos ^{m}\left(\phi-\phi_{0}\right)\right\}$. Such integrals are not found in tables (see, for instance, [2]). Here we give a procedure for evaluating the more general type of integrals

$$
\int_{0}^{2 \pi} \cos ^{n^{\prime}} \phi \sin ^{n^{\prime \prime}} \phi \exp \left\{\sum_{k=1}^{K}\left[a_{k} \cos k\left(\phi-\alpha_{k}\right)+b_{k} \sin k\left(\phi-\beta_{k}\right)\right]\right\} d \phi
$$

(the $a_{k}$ and $b_{k}$ are complex constants, the $\alpha_{k}$ and $\beta_{k}$ are real constants; throughout this paper $k, m, n, h, j$ and $l$, without or with primes, denote integers; $K$ is some positive integer). The method can also be applied when the integrand is the one in (2) multiplied by a function $f(\phi)$ such that

$$
\int_{0}^{2 \pi} f(\phi) e^{i n \phi} d \phi
$$

is known, or when the limits of integration are not 0 and $2 \pi$, or when $n$ is not an integer (in the latter cases the results are much more complicated).

2. Method of Calculation. The procedure is best illustrated with an example. Suppose that we need to calculate

$$
\int_{0}^{2 \pi} \cos n \phi \exp \left\{a \cos (\phi-\alpha)+2 b \cos ^{2}(\phi-\beta)\right\} d \phi .
$$

We use the expression

$$
\exp \left\{\frac{z}{2}\left(t+\frac{1}{t}\right)\right\}=\sum_{k=-\infty}^{\infty} t^{k} I_{k}(z) \quad(t \neq 0)
$$

Received December 20, 1982.

1980 Mathematics Subject Classification. Primary 33A40, 42A16; Secondary 33A20.

Key words and phrases. Integrals involving exponential and trigonometric functions, modified Bessel functions, Fourier coefficients. 
(Eq. 9.6.33 of [1]), which we write for the following cases:

$$
\begin{aligned}
& z=a \cos \alpha, t=e^{i \phi} \quad: \quad e^{a \cos \alpha \cos \phi}=\sum_{k} e^{i k \phi} I_{k}(a \cos \alpha) ; \\
& z=a \sin \alpha, t=-i e^{i \phi} \quad: \quad e^{a \sin \alpha \sin \phi}=\sum_{h}(-i)^{h} e^{i h \phi} I_{h}(a \sin \alpha) ; \\
& z=b \cos 2 \beta, t=e^{2 i \phi} \quad: \quad e^{h \cos 2 \beta \cos 2 \phi}=\sum_{l} e^{2 i l \phi} I_{l}(b \cos 2 \beta) ; \\
& z=b \sin 2 \beta, t=-i e^{2 i \phi} \quad: \quad e^{h \sin 2 \beta \sin 2 \phi}=\sum_{m}(-i)^{m} e^{2 i m \phi} I_{m}(b \sin 2 \beta ;
\end{aligned}
$$

(here, and in the following, $\Sigma_{k}$ denotes the sum from $k=-\infty$ to $k=\infty$, and analogously for $\Sigma_{h}, \Sigma_{l}$, etc.). Now we multiply Eqs. (5) side by side, then multiply both sides of the new equation by $\exp (\operatorname{in} \phi) d \phi$ and integrate from 0 to $2 \pi$. As $I_{-m}(x)=I_{m}(x)$, we obtain

$$
\begin{aligned}
& \int_{0}^{2 \pi} e^{i n \phi} \exp \left\{a \cos (\phi-\alpha)+2 b \cos ^{2}(\phi-\beta)-b\right\} d \phi \\
& =2 \pi \sum_{k, I, m} i^{k+2 l+m+n} I_{k}(a \cos \alpha) I_{k+2 l+2 m+n}(a \sin \alpha) I_{l}(b \cos 2 \beta) I_{m}(b \sin 2 \beta) .
\end{aligned}
$$

The right-hand side can be simplified by applying the addition theorem for Bessel functions (Eq. 7, p. 361 of [3]),

$$
\sum_{n}(-1)^{n} e^{i n \phi} I_{n}(z) I_{n+\nu}(Z)=I_{\nu}(w) e^{i \nu \psi}
$$

where $w=\left(z^{2}+Z^{2}-2 z Z \cos \phi\right)^{1 / 2}$ and $\psi$ is defined by $Z-z \cos \phi=w \cos \psi$, $z \sin \phi=w \sin \psi$. The addition theorem is applied to the $k$ summation and then, after setting $m=j-l$, to the $l$ summation (the latter step is equivalent to replacing $\alpha$ and $\beta$ by $\alpha-\beta$ and 0 , respectively, and multiplying by $e^{i n \beta}$, since the left-hand side of (6) must be invariant if the origin of $\phi$ is changed). We end up with

$$
\begin{gathered}
\int_{0}^{2 \pi} e^{i n \phi} \exp \left\{a \cos (\phi-\alpha)+2 b \cos ^{2}(\phi-\beta)\right\} d \phi \\
=2 \pi e^{h} e^{i n \alpha} \sum_{j} e^{2 i j(\alpha-\beta)} I_{2 j+n}(a) I_{j}(b) .
\end{gathered}
$$

The result we were looking for is the real part of the right-hand side.

The above example clearly shows the procedure to be followed in order to evaluate integrals like (2):

(a) the exponent is written in terms of $\cos k \phi$ and $\sin k \phi$,

(b) the exponential is written as a product of series of $I_{m}$ functions,

(c) the factor multiplying the exponential is written in terms of $\cos n \phi$ and $\sin n \phi$, so that one can consider integrals with $e^{i n \phi}$,

(d) the integration over $\phi$ is carried out, and the result is simplified by making reiterative use of the addition theorem for Bessel functions.

3. Other Applications. The above procedure can be extended in a straightforward way to integrals other than (2), as we said in the introduction. In most cases, however, the addition theorem cannot be applied, so that the result contains a sum over several indices of the product of several Bessel functions. As an example of a 
case where the addition theorem can be applied let us consider the integral

$$
I \equiv \int_{0}^{2 \pi} \phi^{\nu}(2 \pi-\phi)^{\nu} e^{i q \phi} e^{2 b \cos ^{2} \phi} d \phi .
$$

Using (4), this is seen to be equal to

$$
e^{h} \sum_{k} I_{k}(b) \int_{0}^{2 \pi} \phi^{\nu}(2 \pi-\phi)^{\nu} e^{i(2 k+q) \phi} d \phi .
$$

where the integral can be evaluated using Eqs. 7, p. 424 and 9, p. 425 of [2]. The result is

$$
I=\sqrt{\pi}(2 \pi)^{\nu+1 / 2} \Gamma(\nu+1) e^{b+i \pi q} \sum_{k}(2 k+q)^{-\nu-1 / 2} I_{k}(b) J_{\nu+1 / 2}[\pi(2 k+q)] .
$$

As a last example, we derive the following expansion of the error function (Eq. 7.1.1 of [1]) in terms of the Bessel functions $I_{k}$ :

$$
\sqrt{(\pi / 2 v)} \operatorname{erf}(\sqrt{2 v})=-2 e^{-v} \sum_{k}(-1)^{k} I_{k}(v) /\left(4 k^{2}-1\right)
$$

This relationship is arrived at by applying our procedure to the integral

$$
\int_{0}^{\pi} \sin \phi \exp \left(-2 v \cos ^{2} \phi\right) d \phi
$$

División de Física del Sólido

Comisión Nacional de Energía Atómica

Avenida del Libertador 8250

1429 Buenos Aires, Argentina

1. M. Abramowitz \& I. Stegun, eds., Handhook of Mathematical functions, Dover, New York, 1965.

2. I. S. Gradshteyn \& I. M. Ryzhik, Table of Integrals, Series and Products, Academic Press, New York, 1980.

3. (i. N. Watson, A Treatise on the Theory of Bessel Functions, Cambridge Univ. Press, Cambridge, 1952. 